\title{
Reduced PTEN expression predicts relapse in patients with breast carcinoma treated by tamoxifen
}

\author{
Nael Shoman ${ }^{1}$, Shannon Klassen ${ }^{1}$, Andrew McFadden², Miķelis G Bickis³, \\ Emina Torlakovic ${ }^{1, *}$ and Rajni Chibbar ${ }^{1, *}$ \\ ${ }^{1}$ Department of Pathology; ${ }^{2}$ Department of Surgery and ${ }^{3}$ Mathematical Sciences Group, Department of \\ Computer Science, University of Saskatchewan, Saskatoon, SK, Canada
}

\begin{abstract}
Tamoxifen treatment substantially improves the 10-year survival of women with estrogen-receptor (ER)- $\alpha$ positive tumors. However, approximately one-third of all breast cancer patients with ER- $\alpha$-positive tumors progress on antiestrogen therapy. The molecular mechanism(s) involved in antiestrogen-resistant phenotype of breast carcinoma is not completely understood. The PTEN (phosphatase and tensin homolog deleted on chromosome Ten) gene is a novel candidate tumor suppressor that plays an important role in cell cycle regulation and apoptosis by regulating Protein kinase-B/Akt activity. Previous studies have shown that PTEN downregulation in breast cancer is associated with high-grade tumor, distant metastases and poorer diseasefree survival. Decreased PTEN and/or increased protein kinase B/Akt activity in breast cancer cells has recently been associated with resistance to tamoxifen-induced apoptosis. In this study, we have evaluated PTEN expression by immunohistochemistry in $\mathbf{1 0 0}$ tamoxifen-treated ER- $\alpha$-positive breast cancer patients. Reduced PTEN protein expression was associated with shorter relapse-free survival. When stage I patients were analyzed separately, reduced PTEN expression was a strong predictor of both, shorter relapse-free survival and shorter disease-specific survival. An association of reduced PTEN expression with shorter relapse-free survival and disease-specific survival in stage I patients was still observed after stratification by stage, axillary lymph node status, tumor size, grade, and expression of ER- $\alpha$, progesterone receptor, and Her-2/neu. In summary, our results showed a strong association between downregulation of PTEN expression in ER- $\alpha$-positive tumors and failure to tamoxifen treatment.

Modern Pathology (2005) 18, 250-259, advance online publication, 8 October 2004; doi:10.1038/modpathol.3800296
\end{abstract}

Keywords: PTEN; tamoxifen; breast carcinoma

Tamoxifen, a selective estrogen receptor modulator, is standard endocrine treatment for hormone receptorpositive breast cancer, both in the initial adjuvant therapy and as treatment of patients with metastatic disease. However, approximately one-third of patients with estrogen receptor (ER)- $\alpha$-positive tumors are refractory to tamoxifen therapy and approximately one-third of patients who initially respond to tamoxifen develop resistance. ${ }^{1-3}$ Most breast cancers with acquired endocrine resistance retain ER- $\alpha$ expression, which suggests that the loss of ER- $\alpha$ is not a common cause of resistance to endocrine therapy. ${ }^{4}$ The

Correspondence: Dr R Chibbar, MB, BS, PhD, Department of Pathology, Royal University Hospital, University of Saskatchewan, 103 Hospital Drive, Saskatoon, SK, Canada S7N 0W8.

E-mail: rajni.chibbar@saskatoonhealthregion.ca

*These authors contributed equally to the work.

Received 10 May 2004; revised 11 August 2004; accepted 12 August 2004; published online 8 October 2004 mechanisms associated with resistance to tamoxifen therapy are not completely understood..$^{5-7}$

The phosphatase and tensin homolog deleted on chromosome ten (PTEN) is a lipid phosphatase that antagonizes signal transduction downstream of PI-3 (phosphatidylinositol-3) kinase by dephosphorylating phosphatidylinositol-triphosphate (PtdInsP) and suppresses cell growth through the negative regulation of cell cycle and cell survival. ${ }^{8-12}$ Downregulation of PTEN is associated with increased PI-3 kinase activity with subsequently higher levels of $3^{\prime}$-phosphorylated phosphoinositides, which bind to and activate PK-B/Akt. Activated PK-B/Akt promotes cell survival by phosphorylating and modulating the activity of various transcription factors. ${ }^{13,14}$ Recently, it has been demonstrated in vitro that PI-3/Akt pathway modulates ER- $\alpha$ activity and confers tamoxifen resistance to MCF-7 breast cancer cells. ${ }^{15-17}$

PTEN mutations in metastatic prostate cancer are associated with resistance to antiandrogen therapy. ${ }^{18,19}$ 
The prognostic significance of PTEN protein loss in breast cancer is not well established. A few studies have demonstrated reduced PTEN expression in breast cancer and its association with stage, grade, lymph node metastases and steroid receptor status. ${ }^{20-24}$ To date, none of the studies have examined prognostic significance of reduced PTEN protein expression in ER- $\alpha$-positive breast cancer patients and its potential association with unresponsiveness to tamoxifen.

The main objective of this study was to determine whether PTEN protein expression as determined by immunohistochemistry is related to the outcome of the patients who received adjuvant hormonal therapy, tamoxifen. Association of PTEN expression with stage, grade, tumor size, and tumor expression of estrogen receptor-alpha (ER- $\alpha$ ) progesterone receptor (PR), and Her-2/neu expression was also evaluated.

\section{Materials and methods}

\section{Patients}

In all, 100 patients with ER-positive breast cancer, who were treated with antiestrogen, tamoxifen, were randomly selected from database obtained from Saskatoon Cancer Center. 'Relapse' was defined as occurrence of local recurrence, regional lymph node metastases or distant metastases. All the charts were reviewed to obtain relevant clinical information, recurrence, treatment and follow-up. Two pathologists reviewed slides of the primary breast tumor and representative blocks of formalin-fixed/paraffinembedded tissue were obtained from the pathology archives. The tumors were graded according to modified Bloom and Richardson grading criteria.

\section{Immunohistochemistry}

The PTEN, monoclonal mouse anti-human PTEN clone (1:200, Santa Cruz Biotech, Santa Cruz, CA, USA); ER- $\alpha$ clone 6F11 (1:15, Novocastra, Vector labs Burlington, ON, Canada); PR clone 16 (1:100, Novocastra, Vector labs Burlington, ON, Canada); and c-erbB2 (Her-2/neu) polyclonal (1:300, Dako, Massisauga, ON, Canada) were obtained. Immunohistochemistry was performed on the Ventana NEXES automated immunohistochemistry instrument (Ventana Medical systems, Inc, Tucson, AZ, USA) with an avidin-biotin system on $6 \mu \mathrm{M}$ formalin-fixed paraffin-embedded sections from a representative block, containing both normal and tumor tissue. Slides were cut on a superfrost plus slides and baked overnight at $37^{\circ} \mathrm{C}$. When required antigen retrieval was performed with $6 \mathrm{mM}$ citrate $\mathrm{pH} 6.0$ in a microwave oven.

\section{Staining Interpretation}

A semiquantitative scale was used to score the staining intensity for PTEN, ER- $\alpha$, PR, and Her-2/ neu. Expression of ER- $\alpha$ and PR was considered low if less than $50 \%$ cells were positive and negative if less than $10 \%$ of cells were positive. ${ }^{25}$ Expression of Her-2/neu was not considered overexpressed if scored less than $3+$ as described previously. ${ }^{26}$ Since cutoff levels for reduced PTEN expression by using immunohistochemical methods are not defined so far, we have scored both, staining intensity and the percent of positive cells by using $H$-score (histo-score). $H$-score was calculated as follows: $H$-score $=(\% 1+$ cells $\times 1)+(\% 2+$ cells $\times 2)$ $+(\% 3+$ cells $\times 3)$. The mean PTEN score was used as a cutoff point to designate reduced expression. $H$-score PTEN value was categorized for the purpose of summarizing the data for Table 3 .

\section{Axillary Lymph Node Status}

Axillary lymph node status was defined as follows: a score of 0 for absence of axillary lymph node metastases, a score of 1 for 1-3 involved lymph nodes and a score of 2 for 4 or more involved lymph nodes.

\section{Statistical Analysis}

Statistical analyses were conducted with SPSS 11.0 for Windows (SPSS Inc., Chicago, IL, USA), using $\chi^{2}$, Spearman correlation, Fisher's exact (two-tailed $P$ values) and Linear-by-Linear Association $\chi^{2}$-tests to determine the associations between different variables. Cox regression analysis was conducted to estimate relative risks (with 95\% confidence intervals). ${ }^{27}$ Relapse-free survival and disease-specific survival were calculated by the Kaplan-Meier survival estimates and the log-rank test from the date of diagnosis until last contact or relapse (for relapse-free survival) or death from disease (for disease-specific survival). ${ }^{28}$ The level of significance was set at 0.05 .

\section{Results}

Patients' characteristics are summarized in Table 1. The mean patient follow-up was 6 years (range 410). In total, 97 patients had breast carcinomas with histology of invasive ductal carcinoma, while the rest were invasive lobular carcinomas. The presence of axillary lymph node metastases was strongly associated with shorter relapse-free survival $(P<0.0001)$ and shorter disease-specific survival $(P<0.0001)$. Stage was equally significant predictor of relapse-free survival $(P<0.0001)$ and diseasespecific survival $(P<0.0001)$. However, about 50\% of patients with stage I relapsed in approximately 90 months and $30 \%$ of the stage I patients died in the same period. 


\section{PTEN Expression in Invasive Breast Cancer}

The results of immunohistochemical analyses are summarized in Table 2 . In $80 \%$ of the cases, normal breast parenchyma was also present in the biopsy.

Table 1 Patients' characteristics

\begin{tabular}{lc}
\hline & Total \\
\hline Age in years (mean) & $28-93(62)$ \\
Follow-up in months (mean) & $12-120(72)$ \\
Stage & \\
I & 59 \\
II & 36 \\
III & 2 \\
IV & 3 \\
Tumor size (cm) & \\
$\quad<2$ & 61 \\
$2-4$ & 33 \\
$>4$ & 6 \\
Lymph node metastasis & \\
Yes & 33 \\
No & 67 \\
Total & 100 \\
\hline
\end{tabular}

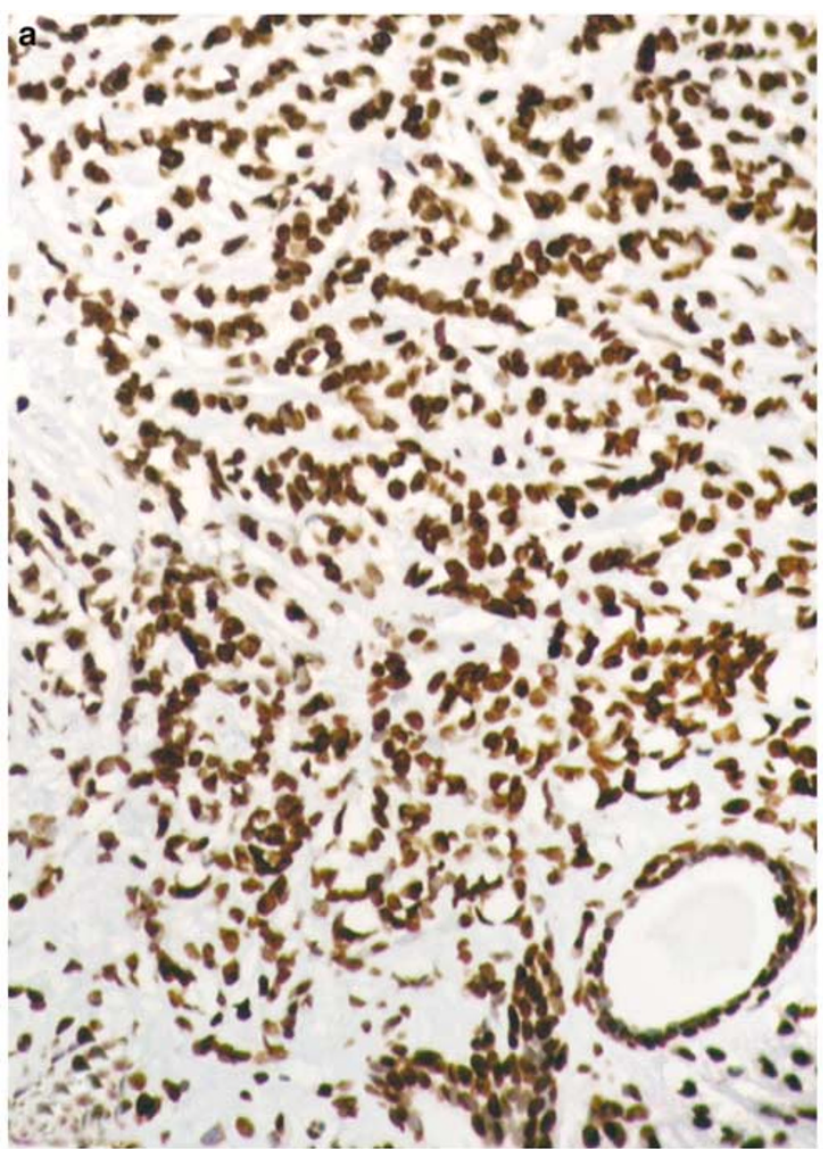

The epithelial cells of normal terminal ducts and lobules showed strong nuclear staining with PTEN antibodies (Figure 1). The intensity and degree of PTEN expression in adjacent neoplastic cells varied considerably. Strong PTEN staining was detected

Table 2 Tumor immunophenotype ${ }^{\mathrm{a}}$

\begin{tabular}{lccc}
\hline & $\begin{array}{c}\text { No } \\
\text { recurrence } \\
(\%)\end{array}$ & $\begin{array}{c}\text { Recurrence } \\
(\%)\end{array}$ & Total \\
& & & \\
\hline $\begin{array}{l}\text { PTEN }(\mathrm{P}<0.0001, \text { FET }) \\
\text { High expression }\end{array}$ & $35(81.4)$ & $25(43.9)$ & $60(60.0)$ \\
Low expression & $8(18.6)$ & $32(56.1)$ & $40(40.0)$ \\
ER- $\alpha(\mathrm{P}=0.227$, FET $)$ & & & \\
High expression & $23(53.5)$ & $23(40.4)$ & $46(46.0)$ \\
Low expression & $20(46.5)$ & $34(59.6)$ & $54(54.0)$ \\
PR $(\mathrm{P}=0.410, F E T)$ & & & \\
High expression & $19(44.2)$ & $20(35.1)$ & $39(39.0)$ \\
Low expression & $24(55.8)$ & $37(64.9)$ & $61(61.0)$ \\
Her-2 $(\mathrm{P}=0.682$, FET $)$ & & & \\
High expression & $24(55.8)$ & $35(61.4)$ & $59(59.0)$ \\
Low expression & $19(44.2)$ & $22(38.6)$ & $41(41.0)$ \\
\hline
\end{tabular}

${ }^{\mathrm{a}}$ High expression $=3+$ and $4+$, low expression $=0-2+$. FET $=$ Fisher's exact test, two-tailed.

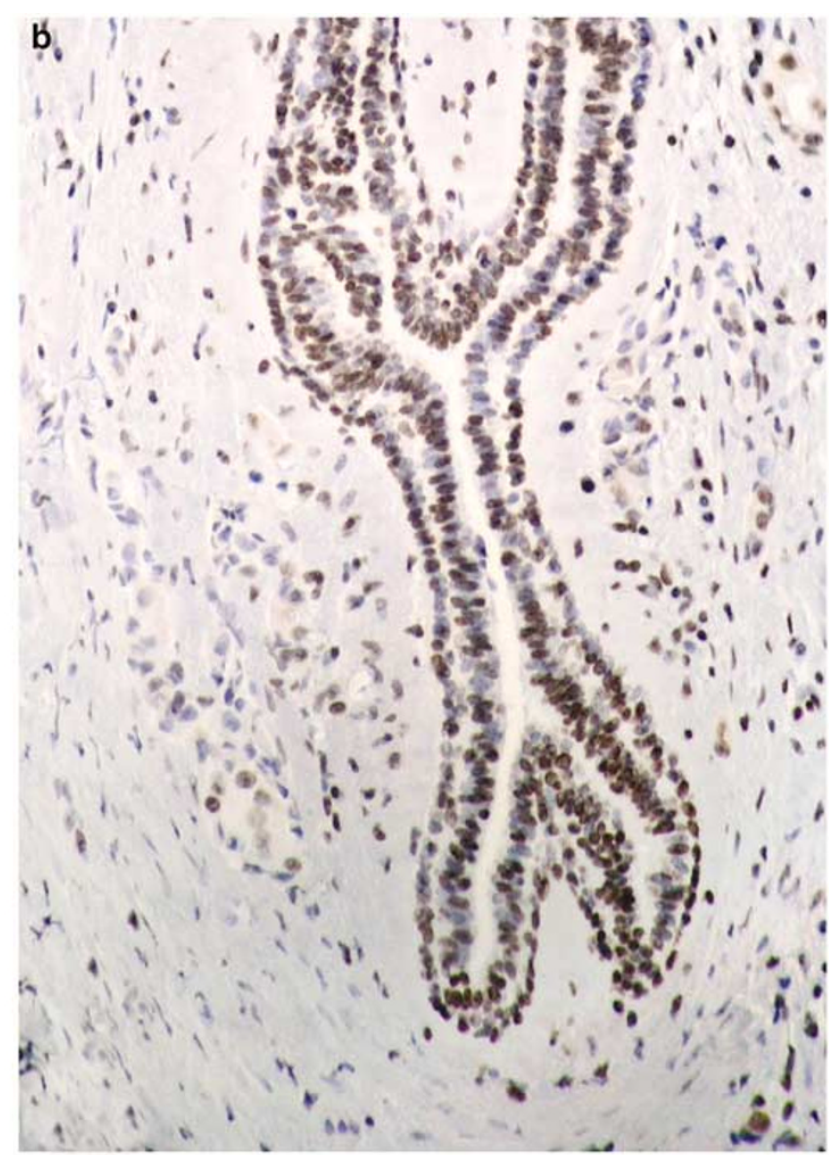

Figure 1 (a) PTEN is strongly expressed in the nuclei of the breast cancer cells as well as in the benign duct epithelial cells (right lower corner). (b) PTEN staining is barely detectable in the neoplastic cells, while strongly expressed in the benign duct epithelial cells. 
in $81 \%$ of nonrecurrence group, while only $43 \%$ in the recurrence group showed similar staining $(P<0.0001$, Fisher's exact test $)$.

PTEN expression was analyzed in relation to clinicopathological parameters including age of the patients, stage, lymph node status, tumor grade, size, and tumor expression of ER- $\alpha$, PR, and Her-2/ $n e u$. No correlation was found between tumor grade and expression of PTEN $(P=0.078$, Spearman correlation, Figure 2a) and no correlation was found for PTEN and PR expression $(P=0.17)$. However, there was a positive correlation between expression of PTEN and ER- $\alpha(P=0.022)$ (Table 3 and Figure $2 b)$. Strong negative association between PTEN expression and the presence of lymph node metastasis was found $(P=0.006)$. In addition, there was no association between PTEN expression and the age of the patients, tumor grade, size, or expression of Her-2/neu. As expected, there was a strong positive association between ER- $\alpha$ and PR expression $(P<0.0001)$. Negative correlation between ER- $\alpha$ expression and the size of the tumor $(r=-0.24$, $P=0.016)$ was noted. PR expression also had negative association with Her-2/neu $(P=0.008)$. Her-2/neu expression was positively associated with the presence of axillary lymph node metastases $(P=0.004)$. The presence of axillary lymph node metastases was associated with higher grade $(P=0.017)$ and larger size of the tumor $(P=0.017)$. Stage and grade were also positively associated $(P=0.002)$ as well as stage and the size of the tumor $(r=0.47, P<0.0001)$.

\section{PTEN Expression, Relapse-Free Survival and Disease-Specific Survival}

Univariate analysis

Reduced expression of PTEN was significantly associated with shorter relapse-free survival $(P=0.0015)$ and there was a trend for shorter disease-specific survival $(P=0.0636)$ (Figure 3$)$. Most significant observation of this study was that the reduced expression of PTEN was associated with both, shorter relapse-free survival $(P=0.0003)$ and shorter disease-specific survival $(P=0.0136)$ in stage I patients (Figure 4). When patients were stratified by high vs low levels $(\geq 50 \%$ cells positive vs $<50 \%$ cells positive) of ER- $\alpha$ expression, reduced
PTEN expression was again associated with less favorable outcome in both groups of patients (Figure 5 ). In stage I patients with low expression of ER- $\alpha$
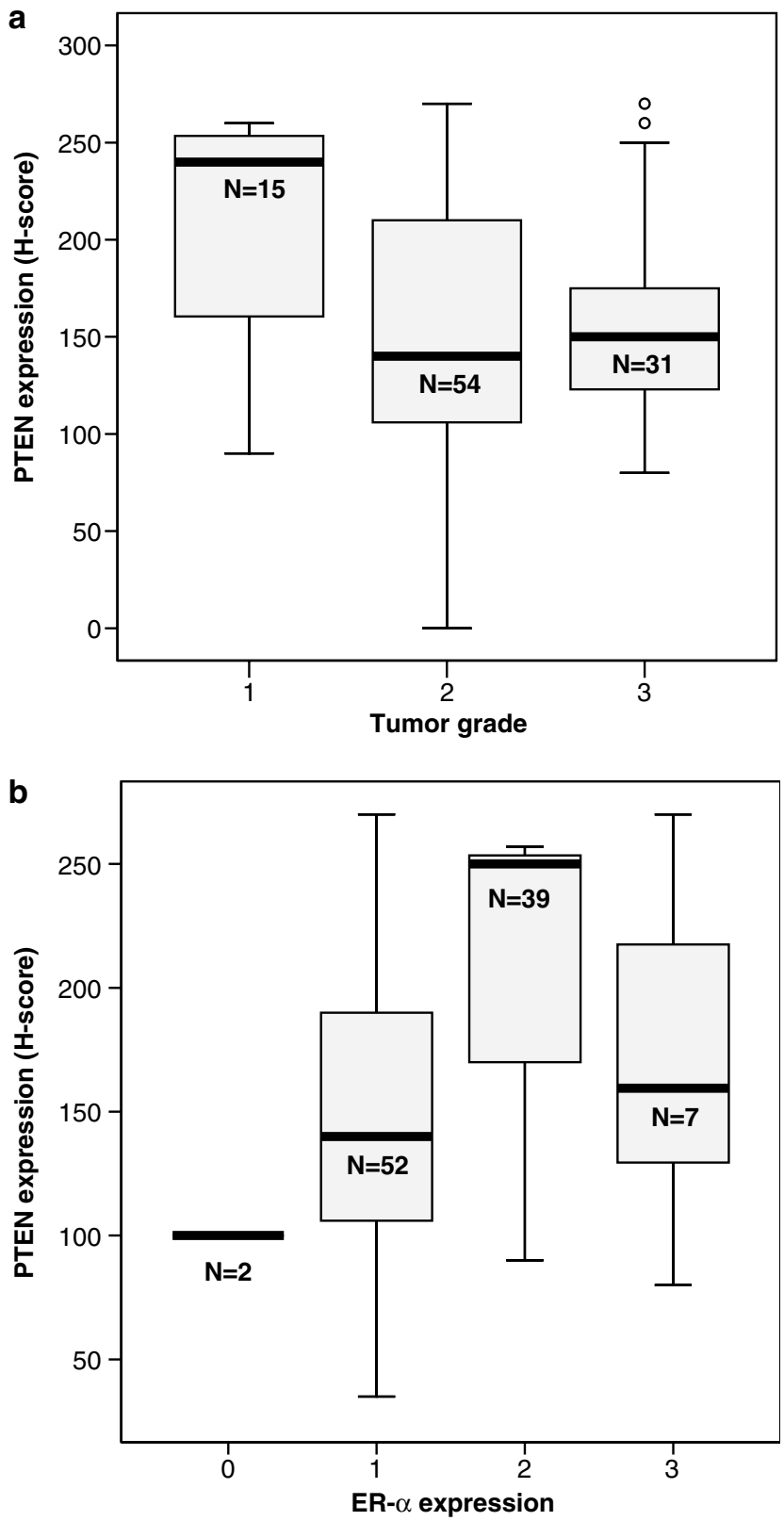

Figure 2 Distribution of PTEN scores according to tumor grade (a) and estrogen receptor- $\alpha$ expression (b).

Table 3 PTEN expression has positive correlation with ER- $\alpha$ expression $(P=0.022$, Spearman correlation)

\begin{tabular}{|c|c|c|c|c|c|}
\hline \multirow[t]{2}{*}{ PTEN } & \multicolumn{4}{|c|}{$E R-\alpha(\%)$} & \multirow[t]{2}{*}{ Total (\%) } \\
\hline & 0 & $1+$ & $2+$ & $3+$ & \\
\hline $1+$ & $0(0.0)$ & 7 (13.5) & $0(0.0)$ & $0(0.0)$ & 7 (7.0) \\
\hline $2+$ & $2(100.0)$ & 18 (34.6) & $2(28.6)$ & $11(28.2)$ & $33(33.0)$ \\
\hline $3+$ & $0(0.0)$ & $16(30.8)$ & $2(28.6)$ & $18(46.2)$ & 36 (36.0) \\
\hline $4+$ & $0(0.0)$ & $11(21.2)$ & 3 (42.9) & $10(25.6)$ & $24(24.0)$ \\
\hline Total (\%) & $2(100.0)$ & $52(100.0)$ & 7 (100.0) & 39 (100.0) & $100(100.0$ \\
\hline
\end{tabular}



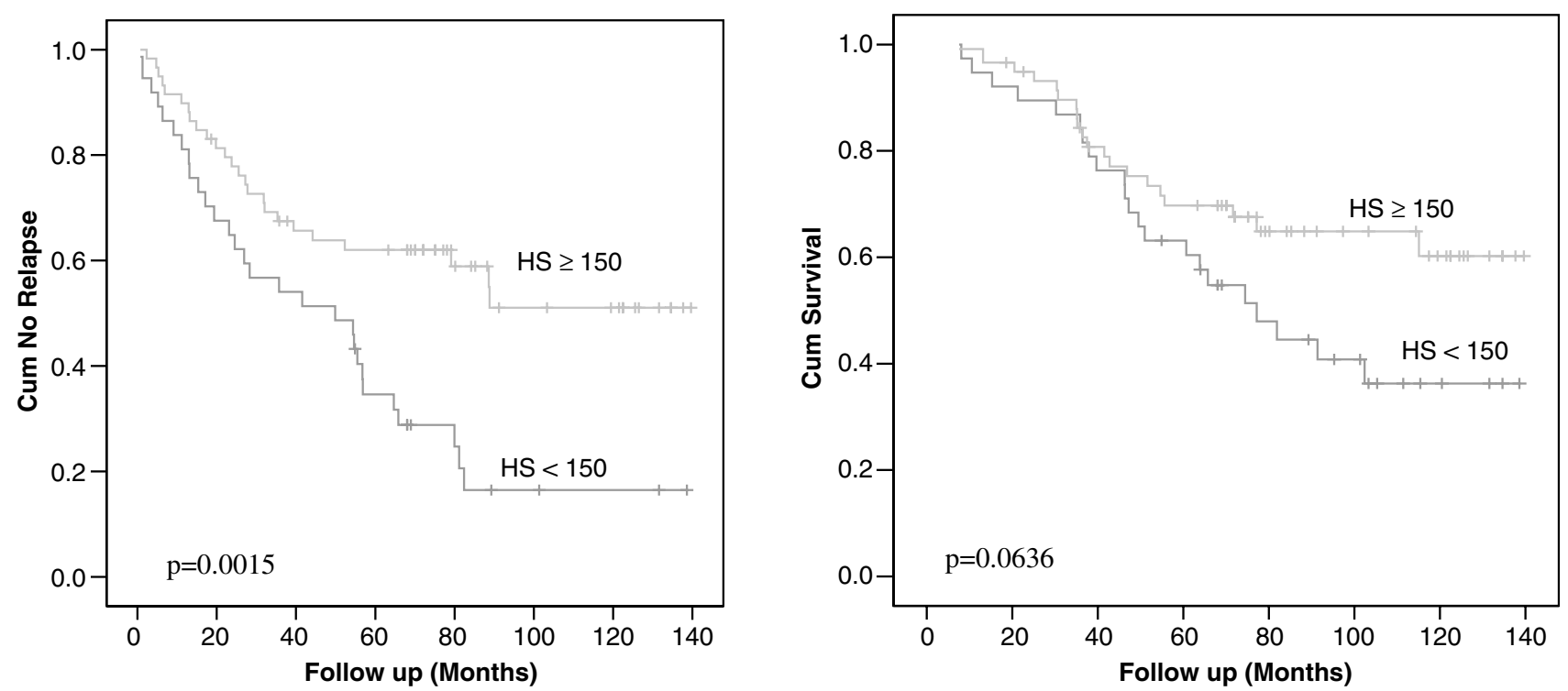

Figure 3 Patients with tumors that do not show downregulation of PTEN expression have significantly longer relapse-free survival as well as statistical trend for longer disease-specific survival.

Stage 1

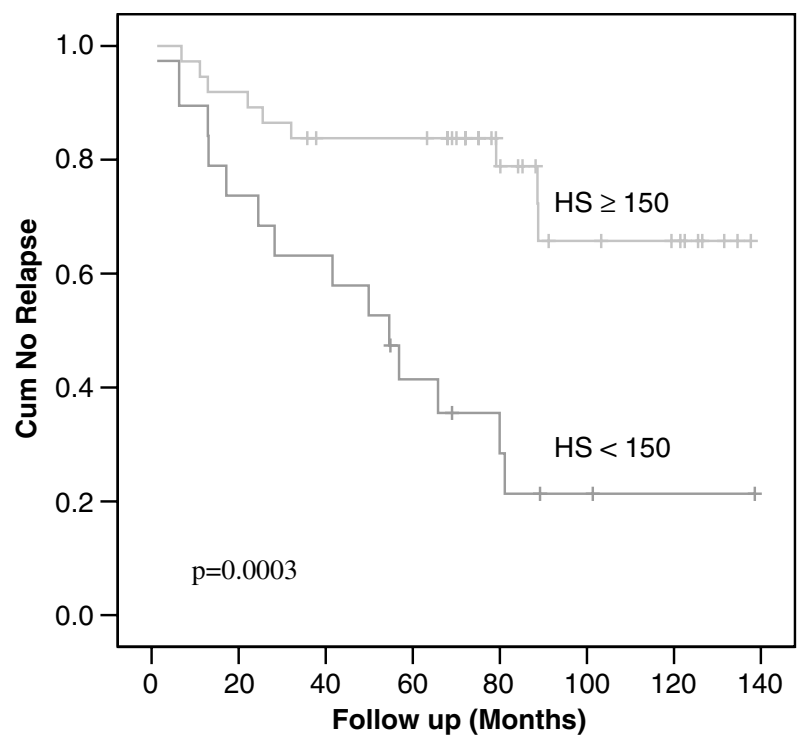

Stage 1

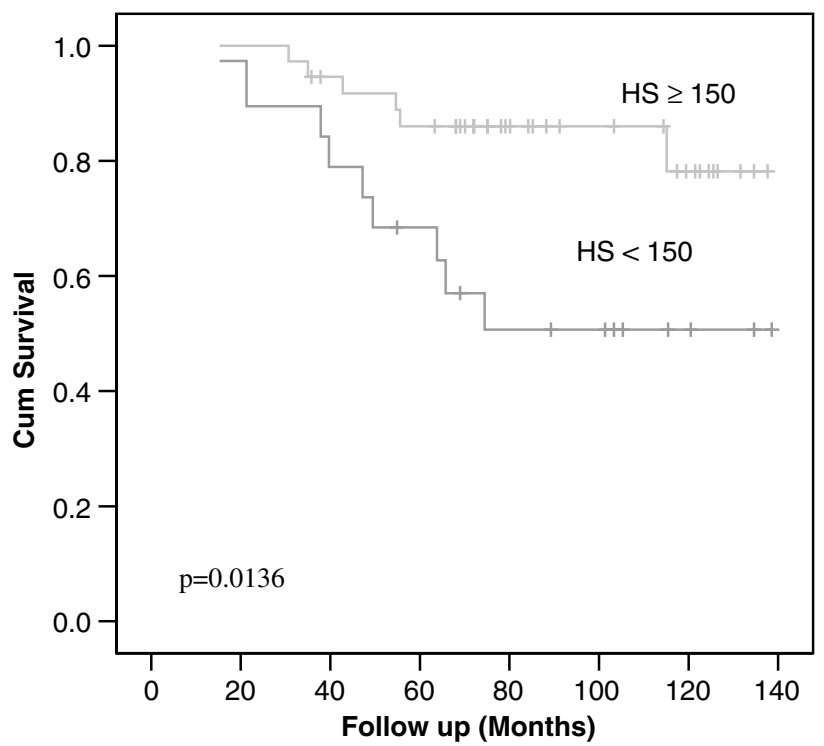

Figure 4 Patients with stage 1 tumors that do not show downregulation of PTEN expression have markedly longer relapse-free survival as well as significantly longer disease-specific survival.

$(<50 \%$ cells positive), the difference in relapse and survival was very large based on PTEN expression. Only $30 \%$ patients relapsed and $25 \%$ died with normal expression of PTEN, while as many as $90 \%$ relapsed and $65 \%$ died if they had tumors with reduced expression of PTEN (Figure 6).

Strong expression of Her-2/neu $(P=0.031)$ was also associated with shorter relapse-free survival. No such association between relapse-free survival was found for the size of the tumor or expression of ER- $\alpha$ or PR. Expression of PR was associated with longer disease-specific survival $(P=0.016)$, while overexpression of Her-2/neu was associated with shorter disease-specific survival $(P=0.020)$. There was no association of disease-specific survival with age of the patients, tumor size, grade, or ER- $\alpha$ expression.

\section{Cox regression analysis}

Cox regression analysis showed that association between reduced expression of PTEN and shorter relapse-free survival was still observed after stratification by stage, tumor size or grade, lymph node 

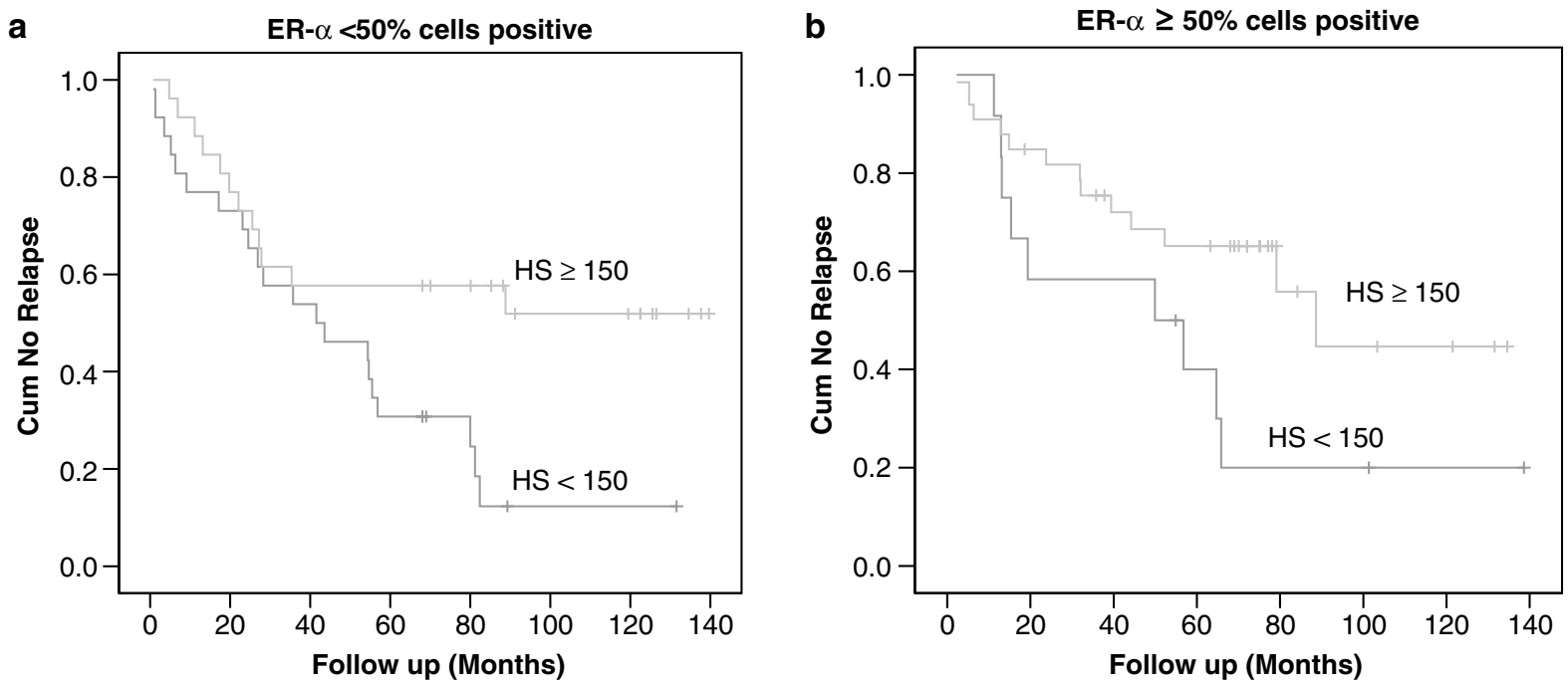

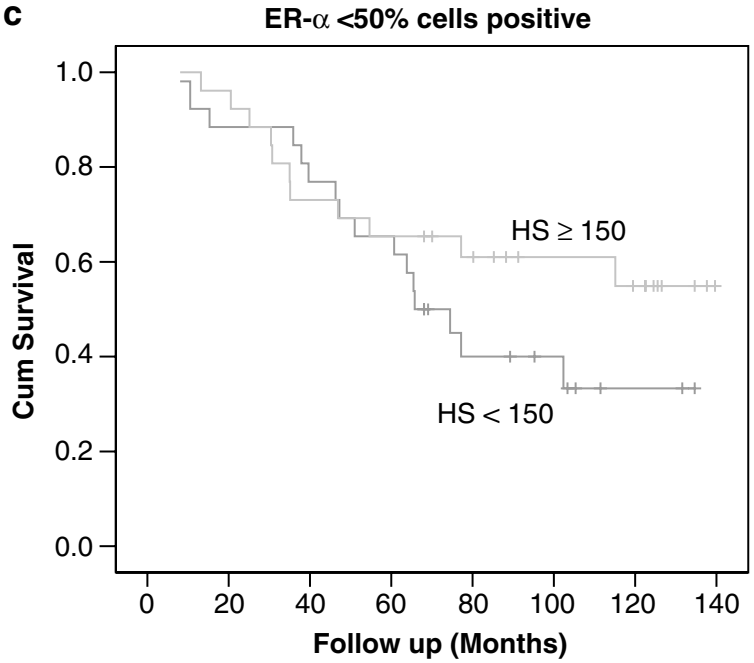

d

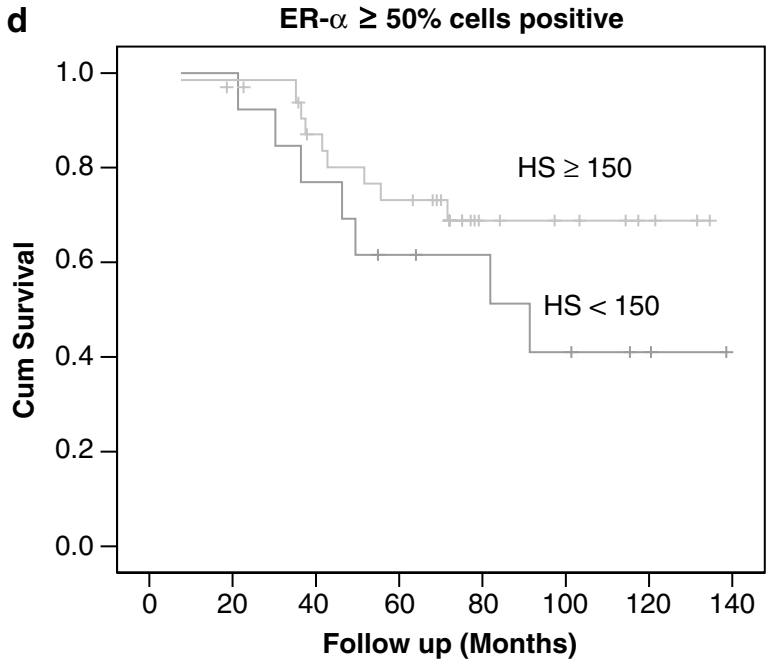

Figure 5 Reduced PTEN expression is associated with shorter relapse-free survival $(P=0.0030)(\mathbf{a})$ and (b) and statistical trend for shorter disease-specific survival (c) and (d) particularly in patients with tumors weakly positive for ER- $\alpha$.

status, and tumor expression of ER- $\alpha$, PR or Her-2/ neu (Table 4).

An association of reduced PTEN expression with shorter relapse-free survival and disease-specific survival in stage I patients was still observed after stratification by tumor size and grade, and tumor expression of ER- $\alpha$, PR, or Her-2/neu (Table 5).

\section{Discussion}

The dual-specificity phosphatase PTEN/MMAC1 has recently been identified as the frequently mutated tumor suppressor gene in various human tumors including endometrial carcinoma, malignant melanoma, high-grade glioma and metastatic prostate cancer. ${ }^{29-33}$ There is an increased predilection for breast cancer caused by germline mutations of PTEN (lifetime risk $>50 \%$ in patients with
Cowden's disease) however, mutations are rare in sporadic breast cancer. ${ }^{34-36}$

Previous studies have demonstrated association between loss of PTEN expression or reduced PTEN expression in breast cancer with stage, lymph node status, disease-related death, and loss of ER. ${ }^{20-24,37}$ Two of these studies included small number of unselected cases. ${ }^{20,24}$ The largest of these studies, also the only follow-up study, included 151 breast cancer cases and showed loss of PTEN expression in $48 \%$ of tumors, significantly higher than in our study. ${ }^{22}$ The difference in the sensitivity of the immunohistochemical analysis may be responsible for this discrepancy. Otherwise, our results in relation to outcome of the patients and association of reduced PTEN expression with adverse clinicopathological variables were similar. All of the studies reported an association between reduced (or loss of) PTEN expression and advanced breast 
a

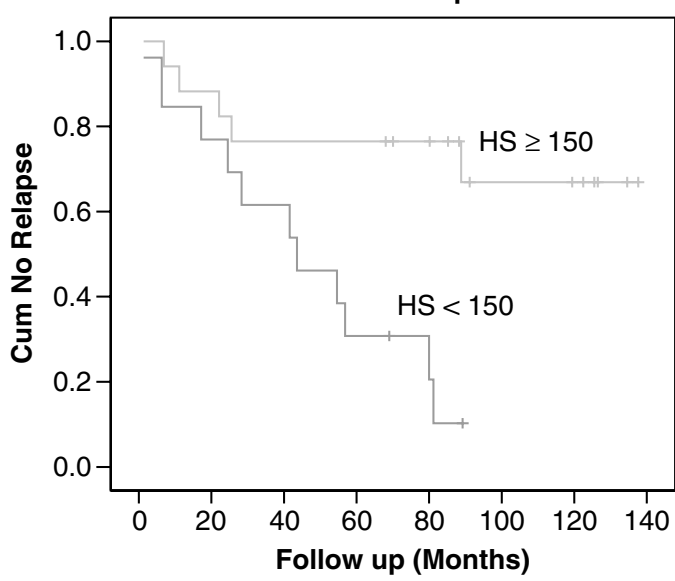

C

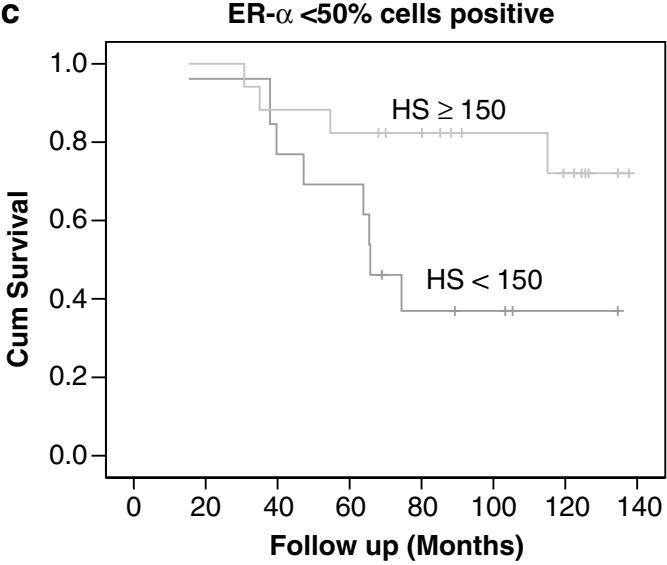

b

ER- $\alpha \geq 50 \%$ cells positive

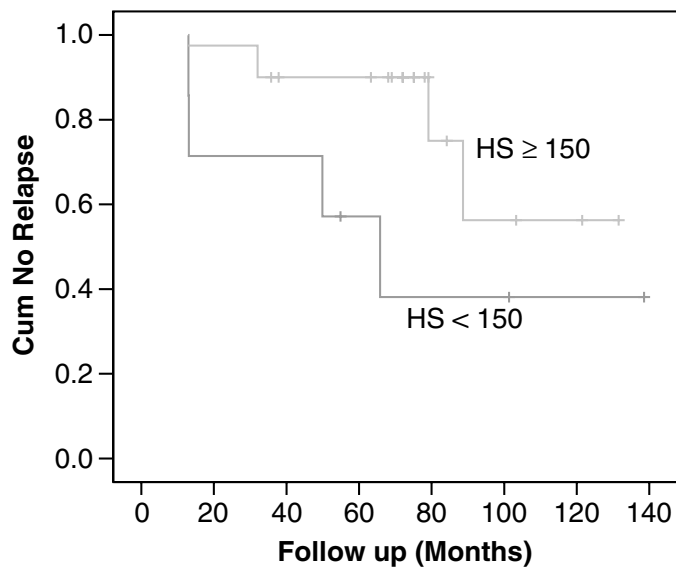

d

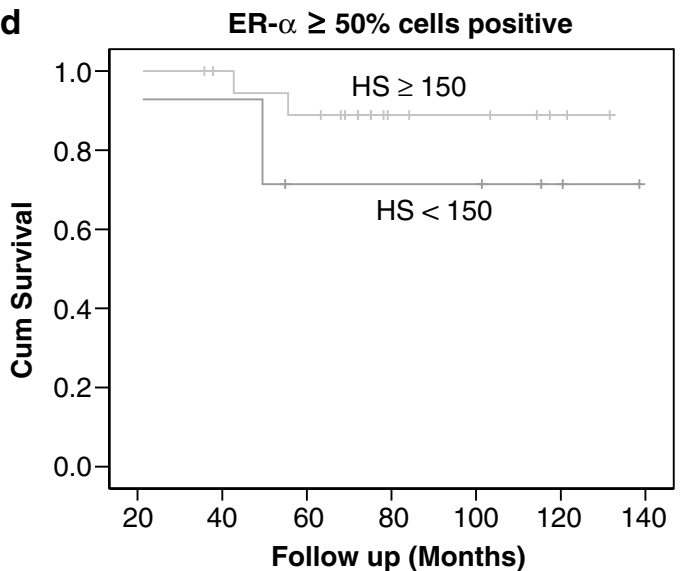

Figure 6 In stage I patients, reduced PTEN expression has even stronger association with shorter relapse-free survival (a,b) and diseasespecific survival $(\mathbf{c}, \mathbf{d})$ particularly in patients with tumors weakly positive for ER- $\alpha(10-50 \%$ positive cells) (a, c).

Table 4 Cox regression analysis estimated prognostic value by hazard ratios (HR) and 95\% CI* for relapse-free survival and diseasespecific survival

\begin{tabular}{|c|c|c|c|c|c|c|}
\hline \multirow[t]{2}{*}{ Variable } & \multicolumn{3}{|c|}{ Relapse-free survival } & \multicolumn{3}{|c|}{ Disease-specific survival } \\
\hline & $\mathrm{P}^{* *}$ & $H R$ & $95 \% C I$ & $\mathrm{P}^{* *}$ & $H R$ & $95 \% C I$ \\
\hline PTEN (per score point) & 0.001 & 0.54 & $0.373-0.785$ & 0.218 & 0.775 & $0.517-1.162$ \\
\hline Age (per year) & 0.204 & 1.02 & $0.992-1.039$ & 0.642 & 1.006 & $0.981-1.032$ \\
\hline Stage & 0.004 & 2.05 & $1.263-3.336$ & 0.032 & 1.830 & $1.055-3.175$ \\
\hline Axillary LN metastases (none vs $1-3$ vs $\geq 4$ ) & 0.032 & 1.61 & $1.043-2.492$ & 0.237 & 1.374 & $0.811-2.328$ \\
\hline Tumor size (per cm) & 0.96 & 1.01 & $0.739-1.376$ & 0.909 & 1.022 & $0.701-1.490$ \\
\hline Tumor grade (per point) & 0.80 & 0.94 & $0.593-1.498$ & 0.886 & 0.962 & $0.566-1.635$ \\
\hline ER- $\alpha$ (per score point) & 0.46 & 1.11 & $0.841-1.469$ & 0.483 & 1.114 & $0.824-1.507$ \\
\hline PR (per score point) & 0.74 & 1.05 & $0.810-1.348$ & 0.196 & 0.826 & $0.618-1.104$ \\
\hline Her-2 (per score point) & 0.04 & 1.23 & $1.008-1.502$ & 0.309 & 1.127 & $0.895-1.420$ \\
\hline
\end{tabular}

${ }^{*}$ CI denotes confidence interval.

${ }^{* *} P$-values were computed from Wald statistics.

cancer stage, suggesting that loss of PTEN expression might have prognostic relevance. Similar to other studies, there was a significant positive association between PTEN expression and ER$\alpha .^{20,22}$ However, our study is unique in that we have evaluated the potential significance of reduced
PTEN expression as a prognostic marker of response to tamoxifen treatment.

In our study, reduced PTEN expression was significantly associated with shorter relapse-free survival in breast cancer patients on tamoxifen treatment and also with both, shorter relapse-free 
Table 5 Stage I patients only: the Cox regression analysis estimated prognostic value by hazard ratios (HR) and $95 \%$ CI* for relapse-free survival and disease-specific survival

\begin{tabular}{|c|c|c|c|c|c|c|}
\hline \multirow[t]{2}{*}{ Variable $^{* *}$} & \multicolumn{3}{|c|}{ Relapse-free survival } & \multicolumn{3}{|c|}{ Disease-specific survival } \\
\hline & $\mathrm{P}^{* * *}$ & $H R$ & $95 \% C I$ & $\mathrm{P}^{* * *}$ & $H R$ & $95 \% C I$ \\
\hline PTEN (per score point) & $<0.0001$ & 0.384 & $0.237-0.622$ & 0.032 & 0.577 & $0.349-0.953$ \\
\hline Age (per year) & 0.055 & 1.038 & $0.999-1.077$ & 0.193 & 1.031 & $0.985-1.079$ \\
\hline Tumor size (per cm) & 0.741 & 1.170 & $0.460-2.975$ & 0.702 & 1.255 & $0.392-4.015$ \\
\hline Tumor grade (per point) & 0.098 & 0.529 & $0.249-1.124$ & 0.211 & 0.560 & $0.226-1.390$ \\
\hline ER- $\alpha$ (per score point) & 0.705 & 1.088 & $0.702-1.687$ & 0.664 & 1.129 & $0.653-1.954$ \\
\hline PR (per score point) & 0.509 & 0.874 & $0.586-1.304$ & 0.073 & 0.608 & $0.353-1.048$ \\
\hline Her-2 (per score point) & 0.005 & 1.585 & $1.153-2.179$ & 0.025 & 1.567 & $1.058-2.319$ \\
\hline
\end{tabular}

${ }^{*}$ CI denotes confidence interval.

$* *$ Constant or linearly dependent covariates stage $=1$ and axillary LN metastases $=0$ excluded from analysis.

$* * * P$-values were computed from Wald statistics.

survival and shorter disease-specific survival for stage I patients. These findings were independent of stage or lymph node status and expression of ER- $\alpha$, PR, or Her-2/neu in tumors. These findings suggest that loss of PTEN may indirectly or directly interfere with cellular effects of tamoxifen.

The PTEN gene encodes a dual specificity phosphatase that opposes the effects of PI-3 kinase by dephosphorylating its lipid products. ${ }^{38}$ These products are required for activation of protein kinase B (PK-B), also known as Akt, a serine/threonine kinase involved in cell growth and survival. ${ }^{39}$ PTEN may thus inhibit carcinogenesis through its inhibitory effect on $\mathrm{PKB} / \mathrm{Akt}-3$ signal transduction pathway. PK-B/Akt-3 inhibits apoptosis, induced by wide variety of agents, and promotes cell cycle progression. ${ }^{40}$ Therefore, cells with high Akt-3 levels or reduced PTEN are more resistant to chemotherapeutic agents. ${ }^{15-17,40-43}$ There is a significant negative correlation between low cellular levels of ER- $\alpha$, and PTEN on one side and elevated Akt-3/ PK-B on the other. ${ }^{37,44-46}$ Functional association between PTEN and estradiol is supported by the observation that in knockout mice with a mammaryspecific deletion of the PTEN gene, exposure to estradiol enhanced development of mammary tumor early in life. ${ }^{47}$ It appears that estradiol downregulates PTEN activity by increasing its phosphorylation. ${ }^{48,49}$ In general, estradiol promotes proliferation of mammary epithelial cells and inhibits apoptosis, ${ }^{50}$ which provides the basis for treatment with antiestrogen tamoxifen. ${ }^{51}$ Tamoxifen, a partial ER antagonist, binds to ER- $\alpha$ and mediates its effects via inhibiting the estrogen-dependent activation of AF-2 region. A recent in vitro study showed that in breast cancer cells, increased PI-3 kinase and Akt activate ER- $\alpha$ and protect breast cancer cells from tamoxifen-induced apoptosis. ${ }^{17}$ Campbell et $a l^{17}$ clearly demonstrated a molecular link between activation of the PI-3 kinase/Akt pathway, hormone-independent activation of ER- $\alpha$ and inhibition of tamoxifen-induced apoptosis, and proposed a new cellular mechanism for tamoxifen resistance.
The capacity to overcome mechanisms that promote apoptosis is essential for the development and progression of cancer, and the P1-3 kinase/Akt pathway, which is negatively regulated by PTEN, provides such a mechanism by transmitting a strong survival signal. ${ }^{52,53}$

In our study, ER- $\alpha$-positive breast tumors with reduced PTEN expression had shorter relapse-free survival, which suggests poor response to tamoxifen therapy. The full significance of this finding is not clear as our study is limited by the lack of ER negative and no tamoxifen treatment control. We also did not correlate the level of PTEN protein expression with response to other chemotherapeutic agents as reduced PTEN expression and/or activation of PI 3-K/Akt pathway has been shown to confer resistance to chemotherapy. ${ }^{54-57}$ However, in the light of information from cell culture studies, it may be proposed that reduced PTEN expression can result in increased activity of PK-B/Akt antiapoptotic pathway, which interferes with cellular actions of tamoxifen resulting in tumor recurrence. PTEN overexpression has also been shown to inhibit cell migration, whereas antisense PTEN enhanced cell migration. ${ }^{58,59}$ An association of reduced PTEN expression with the presence of axillary lymph node metastases as well as distant metastases and death from disease suggests that loss of PTEN may also be involved in promotion of the invasive properties of the tumor.

The most significant observation in our study was a very strong association between reduced PTEN protein expression with relapse- and disease-related death in stage I patients. If these findings can be confirmed in future studies, we would like to propose that an attempt to standardize methods for immunohistochemical detection of PTEN should be made, similar to that for Her-2/neu, so that interpretation of the immunostaining may be relevant for clinical practice.

In conclusion, we have found significant association between reduced PTEN protein expression and adverse prognostic factors in breast cancer as well as 
shorter relapse-free survival and disease-specific survival. These results generally concur with previous studies implicating PTEN as a candidate tumor suppressor in breast cancer. Further study of this gene, especially in relevance to tamoxifen response and association with other breast cancer markers, appears warranted.

\section{Acknowledgements}

This work was supported by a grant to Drs R Chibbar and AW McFadden from the Royal University Hospital Foundation, Saskatoon, Saskatchewan.

\section{References}

1 Nicholson RI, Gee JM, Knowlden J, et al. The biology of antihormone failure in breast cancer. Breast Cancer Res Treat 2003;80(Suppl 1):S29-S34.

2 Kurebayashi J. Endocrine-resistant breast cancer: underlying mechanisms and strategies for overcoming resistance. Breast Cancer 2003;10:112-119.

3 Barker S. Anti-estrogens in the treatment of breast cancer: current status and future directions. Curr Opin Investig Drugs 2003;4:652-657.

4 Johnston SR. Acquired tamoxifen resistance in human breast cancer-potential mechanisms and clinical implications. Anticancer Drugs 1997;8:911-930.

5 Schiff R, Massarweh S, Shou J, et al. Breast cancer endocrine resistance: how growth factor signaling and estrogen receptor coregulators modulate response. Clin Cancer Res 2003;9:447S-454S.

6 Dorssers LC, Van der Flier S, Brinkman A, et al. Tamoxifen resistance in breast cancer: elucidating mechanisms. Drugs 2001;61:1721-1733.

7 Johnston SR, Head J, Pancholi S, et al. Integration of signal transduction inhibitors with endocrine therapy: an approach to overcoming hormone resistance in breast cancer. Clin Cancer Res 2003;9:524S-532S.

8 Besson A, Robbins SM, Yong VW. PTEN/MMAC1/ TEP1 in signal transduction and tumorigenesis. Eur J Biochem 1999;263:605-611.

9 Cantley LC, Neel BG. New insights into tumor suppression: PTEN suppresses tumor formation by restraining the phosphoinositide 3-kinase/AKT pathway. Proc Natl Acad Sci USA 1999;96:4240-4245.

10 Mutter GL. Pten, a protean tumor suppressor. Am J Pathol 2001;158:1895-1898.

11 Myers Jr MP, Pass I, Batty IH, et al. The lipid phosphatase activity of PTEN is critical for its tumor suppressor function. Proc Natl Acad Sci USA 1998; 95:13513-13518.

$12 \mathrm{Wu} \mathrm{X}$, Senechal K, Neshat MS, et al. The PTEN/ MMAC1 tumor suppressor phosphatase functions as a negative regulator of the phosphoinositide 3-kinase/ Akt pathway. Proc Natl Acad Sci USA 1998;95: 15587-15591.

13 Datta SR, Brunet A, Greenberg ME. Cellular survival: a play in three Akts. Genes Dev 1999;13:2905-2927.

14 Stambolic, V, Suzuki A, De la Pompa JL, et al. Negative regulation of $\mathrm{PKB} / \mathrm{Akt}$-dependent cell survival by the tumor suppressor PTEN. Cell 1998;95:29-39.
15 Faridi J, Wang L, Endemann G, et al. Expression of constitutively active Akt-3 in MCF-7 breast cancer cells reverses the estrogen and tamoxifen responsivity of these cells in vivo. Clin Cancer Res 2003;9: 2933-2939.

16 Clark AS, West K, Streicher S, et al. Constitutive and inducible Akt activity promotes resistance to chemotherapy, trastuzumab, or tamoxifen in breast cancer cells. Mol Cancer Ther 2002;1:707-717.

17 Campbell RA, Bhat-Nakshatri P, Patel NM, et al. Phosphatidylinositol 3-kinase/AKT-mediated activation of estrogen receptor alpha: a new model for anti-estrogen resistance. J Biol Chem 2001;276: 9817-9824.

18 Nan B, Snabboon T, Unni E, et al. The PTEN tumor suppressor is a negative modulator of androgen receptor transcriptional activity. J Mol Endocrinol 2003;31:169-183.

19 Ghosh PM, Malik S, Bedolla R, et al. Akt in prostate cancer: possible role in androgen-independence. Curr Drug Metab 2003;4:487-496.

20 Perren A, Weng LP, Boag AH, et al. Immunohistochemical evidence of loss of PTEN expression in primary ductal adenocarcinomas of the breast. Am J Pathol 1999;155:1253-1260.

21 Liu F, Shi W, Xhang XH, et al. Relevance of the PTEN$\mathrm{PKB}$ pathways in human lymph node negative breast cancer. Int J Rad Onc Biol Phys 2001;51:191-196.

22 Depowski PL, Rosenthal SI, Ross JS. Loss of expression of the PTEN gene protein product is associated with poor outcome in breast cancer. Mod Pathol 2001;14: 672-676.

23 Chung MJ, Jung SH, Lee BJ, et al. Inactivation of the PTEN gene protein product is associated with the invasiveness and metastasis, but not angiogenesis, of breast cancer. Pathol Int 2004;54:10-15.

24 Bose S, Crane A, Hibshoosh H, et al. Reduced expression of PTEN correlates with breast cancer progression. Hum Pathol 2002;33:405-409.

25 Rhodes A, Jasani B, Barnes DM, et al. Reliability of immunohistochemical demonstration of oestrogen receptors in routine practice: interlaboratory variance in the sensitivity of detection and evaluation of scoring systems. J Clin Pathol 2000;53:125-130.

26 Birner P, Oberhuber G, Stani J, et al, Austrian Breast \& Colorectal Cancer Study Group. Evaluation of the United States Food and Drug Administration-approved scoring and test system of HER-2 protein expression in breast cancer. Clin Cancer Res 2001; 7:1669-1675.

27 Cox DR. Regression models and life-tables. J R Stat Soc [B] 1972;34:187-220.

28 Kaplan EL, Meier P. Non-parametric estimation from incomplete observation. J Am Stat Assoc 1958;53: 457-481.

29 Sulis ML, Parsons R. PTEN: from pathology to biology. Trends Cell Biol 2003;13:478-483.

30 Mutter GL, Lin MC, Fitzgerald JT, et al. Altered PTEN expression as a diagnostic marker for the earliest endometrial precancers. J Natl Cancer Inst 2000;92: 924-930.

$31 \mathrm{Li}$ J, Yen C, Liaw D, et al. PTEN, a putative protein tyrosine phosphatase gene mutated in human brain, breast, and prostate cancer. Science 1997;275: 1943-1947.

32 McMenamin ME, Soung $\mathrm{P}$, Perera S, et al. Loss of PTEN expression in paraffin-embedded primary 
prostate cancer correlates with high Gleason score and advance stage. Cancer Res 1999;59:4291-4296.

33 Poetsch M, Dittberner T, Woenckhaus C. PTEN/ MMAC1 in malignant melanoma and its importance for tumor progression. Cancer Genet Cytogenet 2001; 125:21-26.

34 Garcia JM, Silva JM, Dominguez G, et al. Allelic loss of the PTEN region (10q23) in breast carcinomas of poor pathophenotype. Breast Cancer Res Treat 1999;57: 237-243.

35 Nelen MR, Kremer H, Konings IB, et al. Novel PTEN mutations in patients with Cowden disease: absence of clear genotype-phenotype correlations. Eur J Hum Genet 1999;7:267-273.

36 Freihoff D, Kempe A, Beste B, et al. Exclusion of a major role for the PTEN tumour-suppressor gene in breast carcinomas. Br J Cancer 1999;79:754-758.

37 Shi W, Zhang X, Pintilie M, et al. Dysregulated PTEN$\mathrm{PKB}$ and negative receptor status in human breast cancer. Int J Cancer 2003;104:195-203.

38 Maehama T, Dixon JE. PTEN: a tumour suppressor that functions as a phospholipid phosphatase. Trends Cell Biol 1999;9:125-128.

39 Weng L, Brown J, Eng C. PTEN induces apoptosis and cell cycle arrest through phosphoinositol-3-kinase/ Akt-dependent and -independent pathways. Hum Mol Genet 2001;10:237-242.

40 Fresno Vara JA, Casado E, de Castro J, et al. PI3K/Akt signalling pathway and cancer. Cancer Treat Rev 2004;2:193-204.

41 Viniegra JG, Losa JH, Sanchez-Arevalo VJ, et al. Modulation of PI3K/Akt pathway by E1a mediates sensitivity to cisplatin. Oncogene 2002;21:7131-7136.

42 Simstein R, Burow M, Parker A, et al. Apoptosis, chemoresistance, and breast cancer: insights from the MCF-7 cell model system. Exp Biol Med (Maywood) 2003;228:995-1003.

43 Brognard J, Clark AS, Ni Y, et al. Akt/protein kinase B is constitutively active in non-small cell lung cancer cells and promotes cellular survival and resistance to chemotherapy and radiation. Cancer Res 2001;61: 3986-3997.

44 Perez-Tenorio G, Stal O. Activation of $\mathrm{AKT} / \mathrm{PKB}$ in breast cancer predicts a worse outcome among endocrine treated patients. Br J Cancer 2002;86:540-545.

45 Nakatani K, Thompson DA, Barthel A, et al. Upregulation of Akt3 in estrogen receptor-deficient breast cancers and androgen-independent prostate cancer lines. J Biol Chem 1999;274:21528-21532.

46 DeGraffenried LA, Friedrichs WE, Fulcher L, et al. Eicosapentaenoic acid restores tamoxifen sensitivity in breast cancer cells with high Akt activity. Ann Oncol 2003;14:1051-1056.
47 Li Y, Podsypanina K, Liu X, et al. Deficiency of Pten accelerates mammary oncogenesis in MMTV-Wnt-1 transgenic mice. BMC Mol Biol 2001;2:2.

48 Guzeloglu-Kayisli O, Kayisli UA, Al-Rejjal R, et al. Regulation of PTEN (phosphatase and tensin homolog deleted on chromosome 10) expression by estradiol and progesterone in human endometrium. J Clin Endocrinol Metab 2003;88:5017-5026.

49 Dery MC, Leblanc V, Shooner C, et al. Regulation of Akt expression and phosphorylation by 17beta-estradiol in the rat uterus during estrous cycle. Reprod Biol Endocrinol 200;1:47.

50 Russo J, Hasan Lareef M, Balogh G, et al. Estrogen and its metabolites are carcinogenic agents in human breast epithelial cells. J Steroid Biochem Mol Biol 2003; 87:1-25.

51 Gallo MA, Kaufman D. Antagonistic and agonistic effects of tamoxifen: significance in human cancer. Semin Oncol 1997;24(Suppl 1):S1-71-S1-80.

52 Tamura M, Gu J, Danen EH, et al. PTEN interactions with focal adhesion kinase and suppression of the extracellular matrix-dependent phosphatidylinositol 3-kinase/Akt cell survival pathway. J Biol Chem 1999;274:20693-20703.

53 Choi Y, Zhang J, Murga C, et al. PTEN, but not SHIP and SHIP2, suppresses the PI3K/Akt pathway and induces growth inhibition and apoptosis of myeloma cells. Oncogene 2002;21:5289-5300.

54 Fahy BN, Schliman M, Virudachalam S, et al. AKT inhibition is associated with chemosensitization in the pancreatic cancer cell line MIA-Pa-Ca-2. Br J Cancer 2003;89:391-397.

55 Kip A, West S, Castillo S, et al. Activation of the PI 3K/ Akt pathway and chemotherapeutic resistance. Drug Resis Update 2002;5:234-248.

56 Grunwald V, DeGraffenried L, Russel D, et al. Inhibitors of mTOR reverse doxorubicin resistance conferred by PTEN status in prostate cancer cells. Cancer Res 2002;62:6141-6145.

57 She QB, Solit D, Basso A, et al. Resistance to gefitinib in PTEN-null HER-overexpressing tumor cells can be overcome through restoration of PTEN function or pharmacologic modulation of constitutive phosphatidylinositol $3^{\prime}$-kinase/Akt pathway signaling. Clin Cancer Res 2003;9:4340-4346.

58 Stewart AL, Mhashilkar AM, Yang XH, et al. PI3 kinase blockade by Ad-PTEN inhibits invasion and induces apoptosis in RGP and metastatic melanoma cells. Mol Med 2002;8:451-461.

59 Raftopoulou M, Etienne-Manneville S, Self A, et al. Regulation of cell migration by the C2 domain of the tumor suppressor PTEN. Science 2004;303: 1179-1181. 